\title{
Towards Ontology-Based Battlefield Information Fusion Framework for Decision Support
}

\author{
Xiaoli Li*, Weihua Li, Youmiao Qu \\ School of Computer Science, Northwestern Polytechnical University, Xi'an 710072, China
}

\begin{abstract}
Thorough awareness of battlefield situation is vital for combat while battlefield information is constantly changing and distributed. The commanders are faced with more complicated and dynamic situations to make decisions than ever before. Ontology technology has received increasing interest in computer science community because of its capability of knowledge representation, sharing, automatic reasoning, and reuse of heterogeneous information from multiple sources. In this paper, we discuss the role of ontology to facilitate high level fusion of battlefield information for decision support.
\end{abstract}

Keywords-Ontology, Battlefield Information, Decision support, JDL, OWL, Fusion

\section{INTRODUCTION}

Commanders at different levels of any types of military organizations require timely and accurate situation awareness of the battlefield so as to make effective decisions and take actions. So how to get the basic meaning of the information is of crucial importance for decision making. The objective of information fusion is to get a general scenario of the entities and their relations of the real world, so as to understand the meaning of the situation. The widely influenced data and information fusion model is Joint Directors of Laboratories (JDL). In the model the process of data and information fusion is related by hierarchy; that is: signal feature assessment (L0), entity assessment (L1), situation assessment (L2), impact assessment (L3), and process assessment (L4). Among which the L0 and L1 level fusion are also called low-level fusion, L2 and L3 level fusion are called high-level fusion. The former has been studied thoroughly during the last decades, getting various theories, algorithms and tools to process multi-sensor signals and to estimate object properties. The ultimate objective of the high-level fusion procedures is to achieve situation assessment; i.e., to understand the scene in terms that can be easily communicated to intelligence officer, to evaluate short and long-term threats, and to support decision making [1].

In computer science ontologies represent a shared understanding of specific domain through classes, properties, and relations. They enable restrictions between entities, interoperability and automatic inference. So ontologies are widely used to represent the situation observed from different sources. As mentioned in[2], "Moreover, ontologies present a growing interest to deal automatically with new kinds of information that begin to exceed military sensor systems, such as web sites, public media, blogs, anonymous tips, direct human sources, which are expressed in natural language. Especially in military operations, critical information comes also from the commander's intent, orders, doctrines and directives, which flow as free text elements. These new kinds of information can be of crucial importance in understanding a situation and predict/evaluate possible threat from a political/ geographical/ etc. context.”

In this paper, we provide an ontology-based framework for battle field knowledge representation and reason-where heterogeneous doctrines, regulations, information sources, reports, commanders and systems are involved. We also present related approaches and existing technologies for highlevel fusion and decision support.

\section{DATA AND INFORMATION FUSION}

\section{A. Related works}

Various data and image from sensors only tell one or some aspects of the measured object, the meaning or the situation of the tested object is not directly given. It is only by the process of comprehension of the trained man that the meaning or situation of the measured data can be given. Professionals can naturally get the meaning of the data or signal associated with their knowledge. How to make the machines automatically get the meaning of the tested data or signal leads the creation of Data and Information Fusion (DIF).

Information as organized data, the relations among entities, attributes, or properties characterized by data are sophisticated. DIF involves a process of combining data and information from multiple inputs, the purpose is to derive enriched information compared to that obtained from each individual input [3]. DIF techniques were first introduced to the research community in the 1990s. The scope of applications that use DIF techniques for problem-solving has extended

\footnotetext{
* Corresponding author at: School of Computer Science, Northwestern Polytechnical University, Xi'an 710072,China.

E-mail address: Lixiaoli886@gmail.com (Xiaoli Li)
} 
tremendously from the military arena at the initial stage to many non-military sectors at present.

\section{B. JDL DIF Model}

The original JDL DIF model in Fig.1. was created by the JDL Data Fusion Group of the United States Department of Defense [4]. It is a hierarchical model with diverse levels of functions.

Every level was relatively independent and facilitating communication, comprehension, coordination and cooperation for data fusion communities to identify and solve problems.

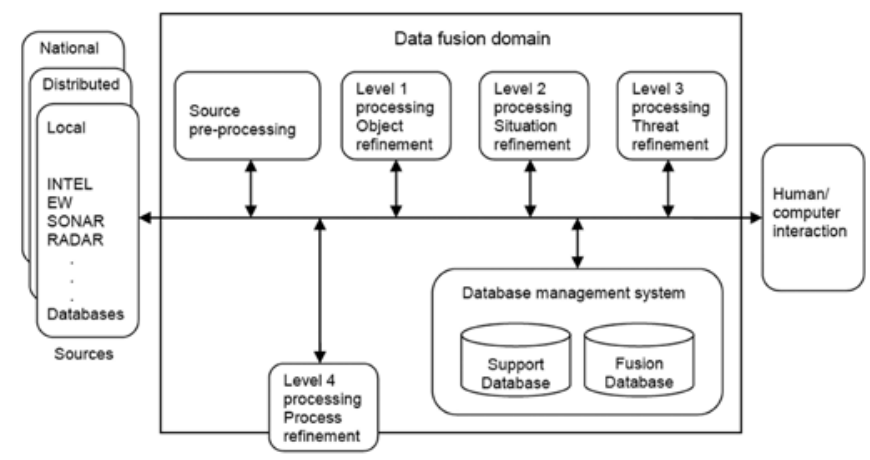

Fig. 1. JDL DIF model [4]

The function of every level was defined as follows:

- Level 0 (Sub-Object Data Assessment)-estimation and prediction of observable states of signals or features;

- Level 1 (Object Assessment)—estimation and prediction of entity states based on data association, as well as continuous and discrete state estimation;

- Level 2 (Situation Assessment)—estimation and prediction of relationships among entities;

- Level 3 (Impact Assessment)-estimation and prediction of effects of entities' actions on goals/missions;

- Level 4 (Process Refinement)—an element of Resource Management that encompasses adaptability in the data collection and fusion processes to support mission objectives;

The Fusion levels were categorized into the low-level fusion process (level 0 and 1) and high-level fusion process (level 2 to 4 ) $[5,6]$.

\section{DFIG Model}

Information Fusion is a comprehensive process including fusion process and management of the platform, sensors. To separate the fusion process and the management, the Data Fusion Information Group (DFIG) proposed a DFIG model [7]. Shown in Fig. 2., include:

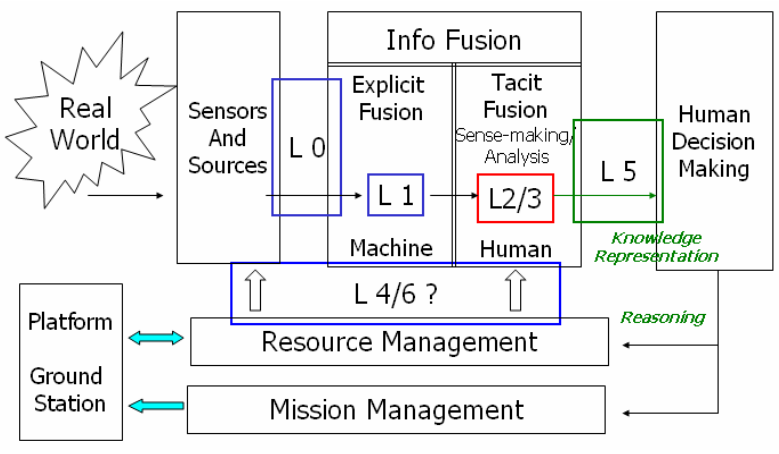

Fig.2. DFIG 2004 model [7]

- Level 0 - Data Assessment: estimation and predication of signal/object observable states on the basis of pixel/signal level data association;

- Level 1- Object Assessment: estimation and predication of entity states on the basis of data association, continuous state estimation and discrete state estimation;

- Level 2 - Situation Assessment: estimation and predication of relations among entities, to include force structure and force relations, communications;

- Level 3 - Impact Assessment: estimation and predication of effects on situations of planned or estimated actions by the participants; to include interactions between action plans or multiple players;

- Level 4 - Process Refinement (an element of Resource Management): adaptive data acquisition and processing to support sensing objectives;

- Level 5 - User Refinement (an element of Knowledge Management): adaptive determination of who queries information and who has access to information and adaptive data retrieved and displayed to support cognitive decision making and actions;

- Level 6 - Mission Management (an element of Platform Management that encompasses adaptability in the determination of spatial-temporal asset control, as well as route planning and goal determination to support team decision making and actions.

The JDL DIF model explains for automatic machine processing, but not for human processing. In actual world, the purpose of DIF is to enhance human user's perception and awareness of his or her environment, and by means of the experience of expert of domain specific to achieve better results, so the user plays an important role in the DIF process [8].

\section{High-level Fusion and Situation Awareness}

After many years of intensive research, low-level data fusion which focus on signal and feature processing has quite matured [9]. Whereas high-level data and information fusion which focus on Situation Awareness (SAW) and inference that lead to better decision making, Impact or Threat Assessment, and Process Refinement or Resource Management attract much research attention [10-12]. 
Compared to the increasingly mature field of low-level DIF, theoretical and practical challenges posed by high-level DIF are more difficult to handle. Contributing factors include the lack of: well-defined spatio- temporal constraints on relevant evidence, well-defined ontological constraints on relevant evidence and suitable models for causality [3].

Level 2 fusion, named as Situation Assessment whose objective is to determine and interpret the relationships among objects. Situation Awareness (SAW) involves the identification and monitoring of various relationships among physical and abstract entities.

Level 3 fusion, named as Impact Assessment whose primary objective includes threats assessment, intents and possibilities evaluation, and uncertainty reducing of battlefield situation awareness.

Contrary to low-level information fusion (LLIF) which is characterized by data and signal processing, object state estimation, the high-level information fusion (HLIF) is characterized by abstract symbolic representation, knowledge sharing, reusing, inference and reasoning.

\section{DIF AND DECISION SUPPORT}

Decision support is to select a most favorable choice from alternative sequence according to current situation and constraints. So the Situation Awareness (SAW) by DIF and constraints (doctrine, rules and procedures) are the basis of decision support elements.

\section{A. OODA Loop and Decision Support}

As an accurate depiction of both behavior and the command and control process, John Boyd's OODA loop model [13, 14] and it's revisions have been widely used in military Command and Control (C2) process.

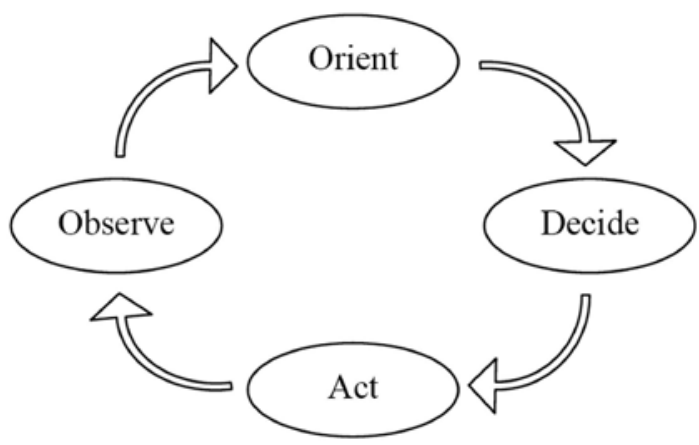

Fig. 3. OODA loop[13]

In Fig. 3., the OODA loop comprises four sequential phases: Observe (gather information from the environment); Orient (gain situation awareness and perform situation/threat assessment based on the information gathered); Decide (respond to situation and work out follow-up actions); and Act (execute the planned respond/action). As the OODA model was directly mapped from the process steps of human in decision making, it lacks clear interface and standard definitions of each steps, e.g. what information need gather, and how to record the gathered information in Observe step, so it lacks the specific guidance in design a computer based system.

The OODA and DFIG models have similar properties in trying to capture the decision process [15]. Fig. 4. Shows a mapping between the DFIG and the OODA loop cyclic process. The traditional information fusion data processing functions (i.e. estimation) include: observe and orient which compose situation awareness. In duality, the information fusion action functions (i.e. control) include: decide and act as the decision making process.

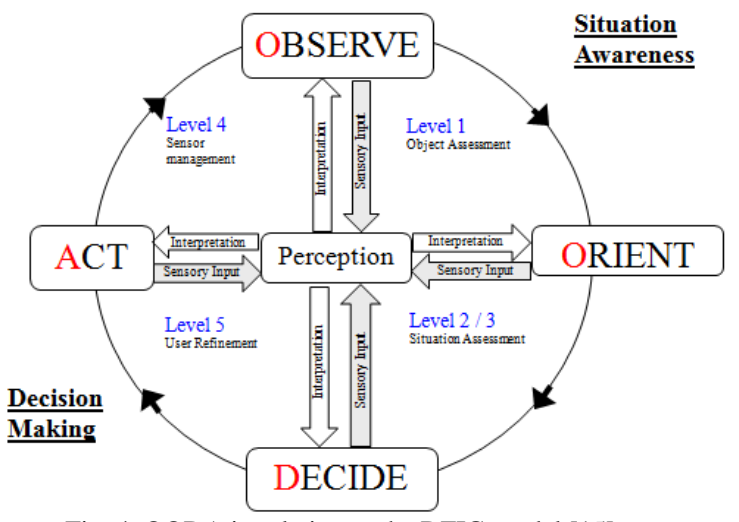

Fig. 4. OODA in relation to the DFIG model [15]

\section{HIGH-LEVEL DIF MODEL FOR BATTLEFIELD}

Commanders in battlefield situations are urgently in need of a thorough grasp of adversary's, friend's and their own force distributions both in temporal and space so as to evaluate possible tactics, chance of combat, assess threat and get hypothesis of adversary's intentions and objectives. Ontologies as an effective means to represent those entities and their relations in computer are naturally introduced in battlefield situation awareness and decision support system.

\section{A. Relations of High-Level Fusion Model}

The key elements for battlefield decision are composed of the analysis of adversary's intentions, combat opportunities, and fight capacity. The intentions consist of attack, retreat and defense et al. The opportunities include temporal and spatial possibilities. The fight capacity is comprised by the quality and quantity of warrior, weapon and logistics et al. The relations among the three key elements are shown in Fig. 5. 


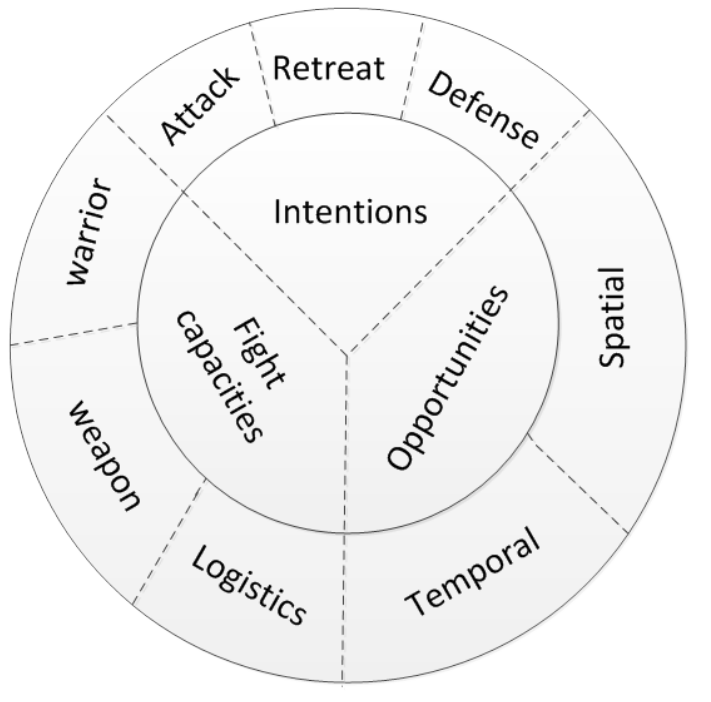

Fig. 5. High-Level Fusion Model

The intentions can be detected by humint and sensors scanning the battlefield for distribution of fight capacity both in spatial and its variety with temporal. In modern combat field, war situations change rapidly, and the transportation speed of warrior, tanks, fighters, missiles et al. varies considerably. An attack at proper spatial in time results in great changes for combat capacities and battlefield situations, so the temporal and spatial information are of vital importance in decision support.

\section{B. Architecture of battlefield decision support system}

Battlefield situation is changing rapidly, modern wars focus not only on the overall fighting force of the contest, but also emphasis on the relative advantages of the local combat force as well as the timely grasping of fighting chance. A variety of tactical means rely on a certain time and a certain battlefield space to form the relative superiority to win the battle. Conversely, commanders clearly knowing the available space and time to effectively changing the relative superiority can save their own strength. Judgment of the intention of the enemy, the choice of the fighting opportunity combined with the expectation of its own fight capacity is important components of battlefield decision-making. Fig. 6. depicts the architecture of battlefield decision support system (DSS).

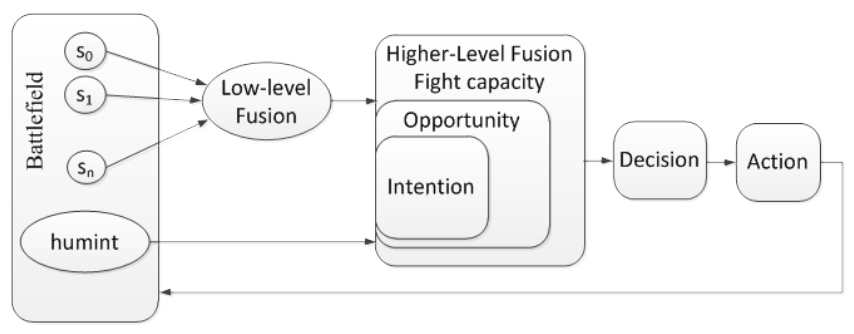

Fig.6. Architecture of Battlefield DSS

Combining the battlefield features obtained by low-level fusion process with humint to perform high-level fusion, commanders can estimate adversaries' intention and make hypotheses, then consulting about temporal and spatial opportunities as well as fight capacity to make decisions.

\section{Acknowledgment}

This research was supported in part by ministries and commissions project. The authors sincerely appreciate the editors for helpful advices and suggestions.

\section{References}

[1]. Bossé, É., A Practical Approach to the Development of Ontology-Based Information Fusion Systems. Prediction and Recognition of Piracy Efforts Using Collaborative Human-Centric Information Systems, 2013. 109: p. 176.

[2]. Bellenger, A., Semantic Decision Support for Information Fusion Applications, 2013, Rouen, INSA.

[3]. Foo, P.H. and G.W. Ng, High-level Information Fusion: An Overview. J. Adv. Inf. Fusion, 2013. 8(1): p. 33-72.

[4]. Hall, D.L. and J. Llinas, An introduction to multisensor data fusion. Proceedings of the Ieee, 1997. 85(1): p. 6-23.

[5]. Ng, G., Intelligent Systems: Fusion, Tracking and Control CSI, Control and Signal Image Processing Series2003: USA: Philadelphia Research Studies Press.

[6]. Klein, L.A., Sensor and data fusion: a tool for information assessment and decision making. Vol. 324. 2004: SPIE press Bellingham^^ eWA WA.

[7]. Llinas, J., et al., Revisiting the JDL data fusion model II, 2004, DTIC Document.

[8]. Blasch, E. Level 5 (User Refinement) issues supporting information fusion management. in Information Fusion, 2006 9th International Conference on. 2006. IEEE.

[9]. Smith, D. and S. Singh, Approaches to multisensor data fusion in target tracking: A survey. Knowledge and Data Engineering, IEEE Transactions on, 2006. 18(12): p. 1696-1710.

[10]. Blasch, E., et al. High level information fusion developments, issues, and grand challenges: Fusion 2010 panel discussion. in Information Fusion (FUSION), 2010 13th Conference on. 2010. IEEE.

[11]. Costa, P.C., et al. High-level fusion: Issues in developing a formal theory. in Information Fusion (FUSION), 2010 13th Conference on. 2010. IEEE.

[12]. Costa, P.C., et al. High-level information fusion with bayesian semantics. in UAI 9th Bayesian Modeling Applications Workshop, Catalina Island, CA. 2012. Citeseer.

[13]. Nilsson, M., Human decision making and information fusion: Extending the concept of decision support. 2007.

[14]. Das, S., High-level data fusion2008: Artech House.

[15]. Blasch, E.P., et al. User information fusion decision making analysis with the C-OODA model. in Information Fusion (FUSION), 2011 Proceedings of the 14th International Conference on. 2011. IEEE. 